Article

\title{
The Regulation and Function of miR-21-FOXO3a-miR-34b/c Signaling in Breast Cancer
}

\author{
Xiangyan Liu ${ }^{1,2}$, Jie Feng ${ }^{3}$, Lili Tang ${ }^{2}$, Liqiu Liao ${ }^{1}$, Qing $X u^{4}$ and Shaihong Zhu ${ }^{1, *}$ \\ 1 Department of General Surgery, the Xiangya Hospital, Central South University, \\ 138 Tongzipo Road, Changsha 410013, China; E-Mails: xiangyanliu@yahoo.com (X.L.); \\ musicjie302@yahoo.com (L.L) \\ 2 Department of Breast Surgery, the Xiangya Hospital, Central South University, \\ 138 Tongzipo Road, Changsha 410013, China; E-Mail: lilitang474@yahoo.com \\ 3 Department of Nursing, Thomas Jefferson University, Philadelphia, PA 19107, USA; \\ E-Mail: jie.feng@jefferson.edu \\ 4 Department of Pathology, Anatomy and Cell Biology, Thomas Jefferson University, Philadelphia, \\ PA 19107, USA; E-Mail: qxwelcome@gmail.com
}

* Authors to whom correspondence should be addressed; E-Mail: zhushaihong@medmail.com.cn; Tel.: +86-731-8861-8476; Fax: +86-731-8861-8471.

Academic Editor: Jens Schlossmann

Received: 7 November 2014 / Accepted: 27 January 2015 / Published: 30 January 2015

\begin{abstract}
Upregulation of miR-21 (microRNA-21) and downregulation of miR-34b/c have been found in breast cancer $(\mathrm{BC})$. However, their regulation mechanism and function roles in $\mathrm{BC}$ have not been fully addressed. Here, we report that miR-21 levels were inversely correlated with miR-34b/c levels in BC. MiR-21 upregulation contributes to PTEN downregulation, which is beneficial for the activation of PI3K/AKT signaling. The activation of AKT phosphorylates FOXO3a, triggering relocalization of FOXO3a proteins from the nucleus to the cytoplasm. FOXO3a is a newly identified transcription factor responsible for miR-34b/c expression. Downregulation of nuclear FOXO3a decreased the expression levels of miR-34b and miR-34c in breast cancer cells, in which p53 was mutated. We also found upregulation of circulating miR-21 and downregulation of circulating miR-34b/c in BC patients' serum. More importantly, we showed that systemic delivery of miR-34b/c or with anti-miR-21 significantly inhibited breast tumor growth in vivo. These results suggest that high circulating levels of miR-21 and low levels of miR-34b/c may provide potential
\end{abstract}


biomarkers for $\mathrm{BC}$ diagnosis, and systemic delivery of miR-34b/c has potential as a therapeutic option for BC treatment.

Keywords: miR-21; miR-34b/c; FOXO3a; breast cancer; miRNAs injection

\section{Introduction}

Breast cancer $(\mathrm{BC})$ is the most common malignant disease in women in western countries and is a leading cause of cancer-related death among women worldwide [1]. The incidence rate of breast cancer is increasing dramatically in China in recent years. Although prognostic miRNAs expression signatures have been defined for some breast carcinomas [2-4], the underlying pathways regulating breast cancer aggressiveness remain poorly understood.

MiRNAs (microRNAs) are small endogenous non-coding RNAs that control target-gene expression at post-transcriptional levels. Many miRNAs are implicated as proto-oncogenes or as tumor suppressors and are aberrantly expressed in various cancer types including breast cancer. Among oncogenic miRNAs, miR-21 is one of the most commonly observed aberrant miRNAs in human cancers. MiR-21 dysregulation was first reported in breast cancer by Iorio [5]. MiR-21 has been reported to promote oncogenesis and progression of various carcinomas via direct targeting of tumor suppressing phosphatase and tensin homolog (PTEN) [6]. Recently, emerging evidences demonstrated that the miR-34 family (miR-34a, miR-34b, and miR-34c) are down-regulated and play an important role as a tumor suppressor in various types of cancers [7-10]. MiR-34a is encoded by its own transcript, whereas miR-34b and miR-34c share a common primary transcript. The miR-34 family directly targets/regulates many proteins involved in various biological processes, such as cell cycle, apoptosis, cell migration and metastasis [11]. Moreover, systemic delivery of miR-34a in a mouse model of lung tumor resulted in reduced tumor burden [12]. The past several years of studies have clearly shown that the miR-21 and miR-34 families are the master regulators of tumor biology. However, the regulation mechanisms and the therapeutic roles of miR-21 and miR-34b/c in breast cancer have not yet been fully clarified. In our study, we demonstrated that (i) miR-21 regulated miR-34b/c through affecting PI3K/AKT/FOXO3a signaling; (ii) circulating miR-21 and miR-34b/c have clinical implication for providing novel biomarkers for $\mathrm{BC}$ diagnosis; and (iii) targeting $\mathrm{miR}-21 \mathrm{and} /$ or restoring miR-34b/c may possess therapeutic applications for BC treatment in the future.

\section{Results and Discussion}

\subsection{Expression of MiR-21 and MiR-34b/c in Breast Cancer Cell Lines, Clinical Specimens, and Serum Samples}

We first conducted a miRNA array analysis to investigate the miRNAs expression profiles in three paired breast carcinoma tissues (BCT) and non-malignant adjacent tissues (NT). We identified several of the most significantly up- and down-regulated miRNAs that have been previously implicated in breast cancer, including miR-21 (up-regulated) and miR-34b/c (down-regulated). We subsequently validated this finding by quantitative RT-PCR (RT-qPCR) for miR-21, miR-34b, and miR-34c in an 
independent group of 15 paired BCT and NT (see Figure 1A). Notably, Spearman's rank correlation analysis showed that the miR-21 level was inversely correlated with miR-34b (correlation $=-0.608$, $p=0.016, n=15$ ) and miR-34c (correlation $=-0.584, p=0.022, n=15$ ) levels in 15 breast cancer specimens (see Figure 1B). Next, we examined the expression levels of these miRNAs in three human $\mathrm{BC}$ cell lines and one immortalized breast epithelial cell line, MCF-10A. Consistent with the data obtained from breast tissue specimens, miR-21 was significantly up-regulated, and miR-34b/c was dramatically down-regulated in all three BC cell lines compared with MCF-10A (see Figure 1C). More interestingly, we found that levels of circulating miR-21 were higher, and circulating miR-34b/c were lower in BC patients' serum than in serum from healthy controls (see Figure 1D), indicating that high circulating miR-21 and low miR-34b/c may serve as potential biomarkers for BC diagnosis.
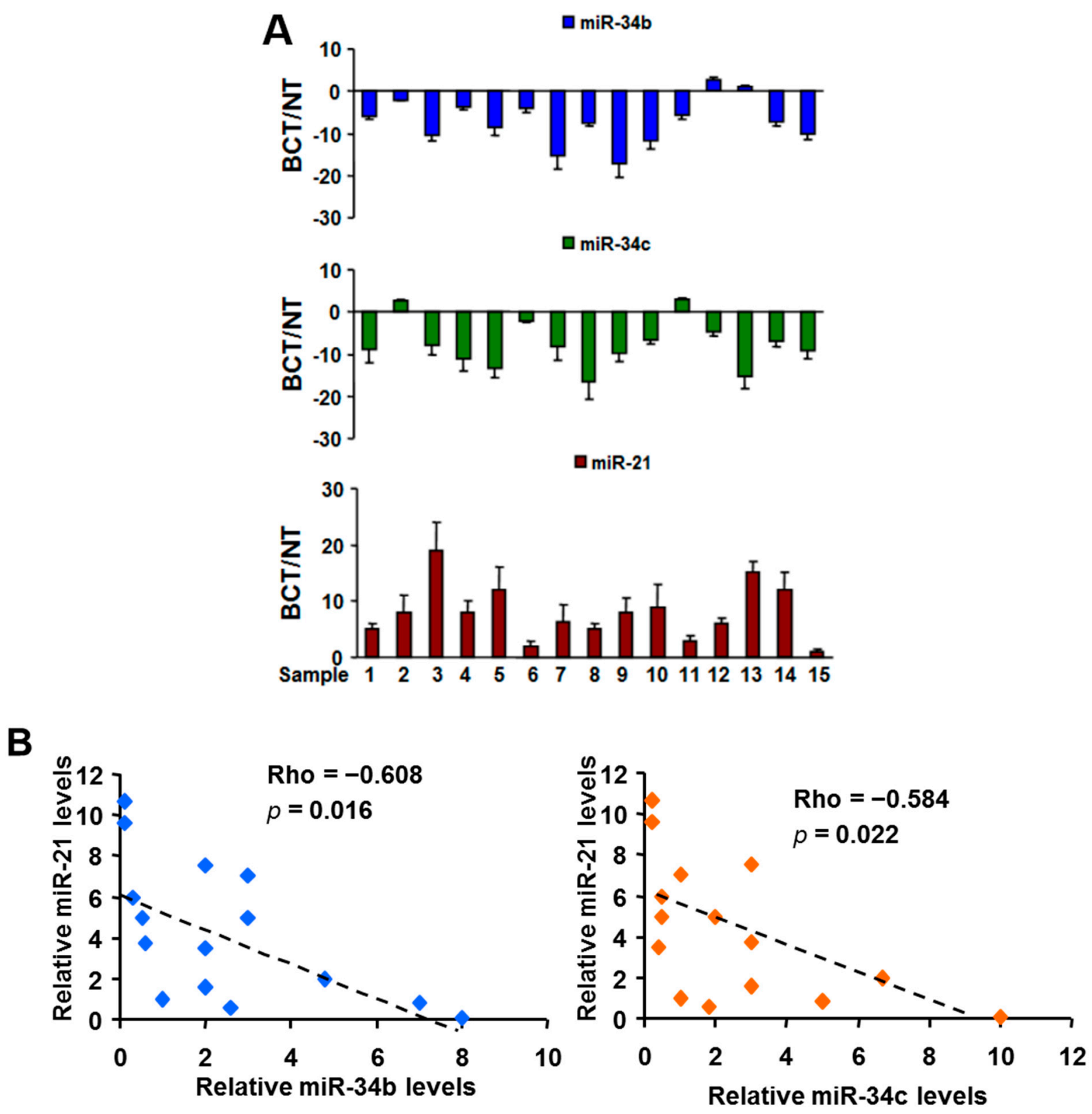

Figure 1. Cont. 

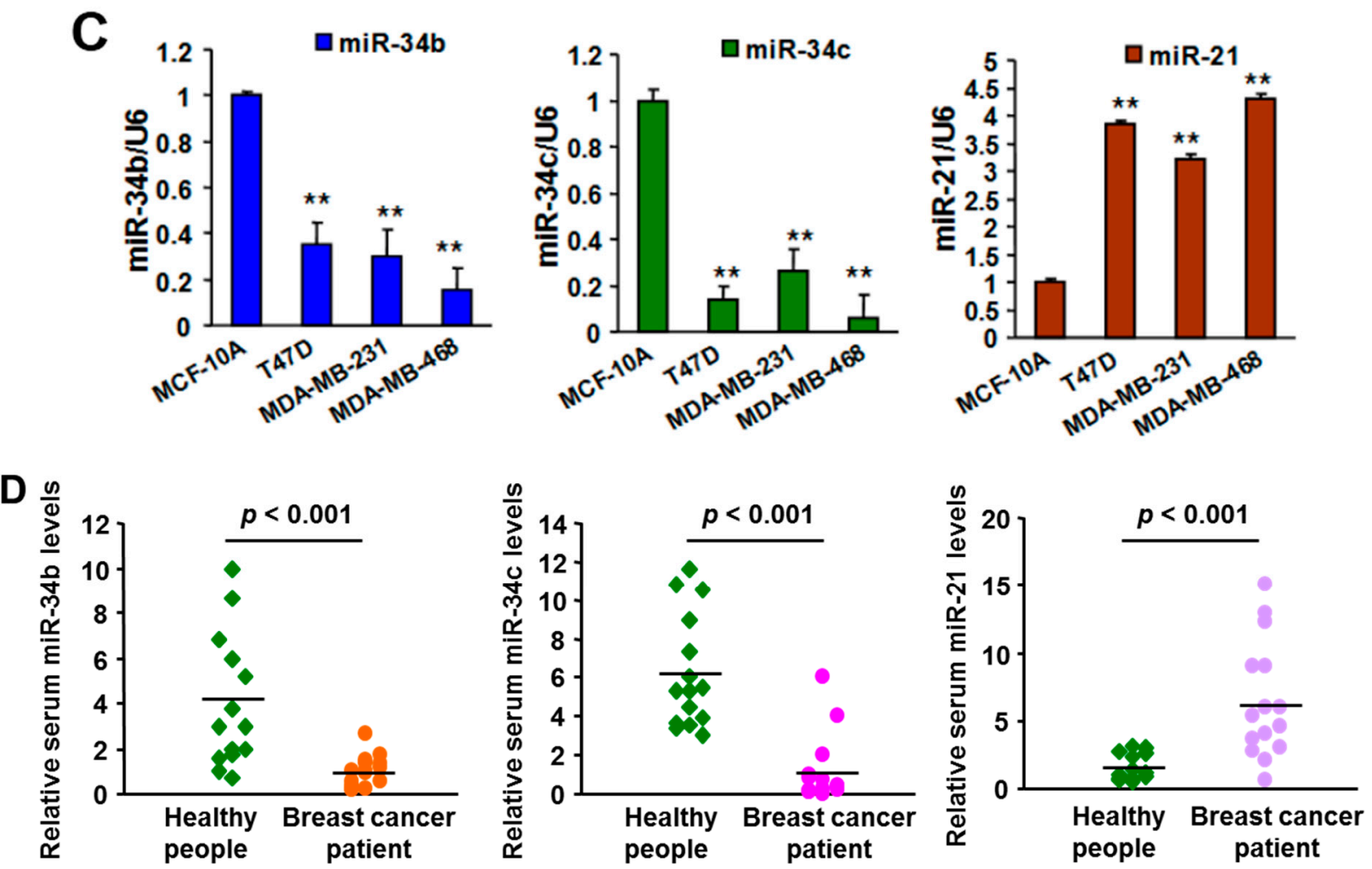

Figure 1. MiR-21 was upregulated and $\mathrm{miR} 34 \mathrm{~b} / \mathrm{c}$ was down-regulated in breast cancer cells, tissues, and serums. (A) Relative expression levels of miR-21, miR-34b and miR-34c in 15 paired of breast cancer tumors (BCT) and non-malignant adjacent tissues (NT) were determined by RT-qPCR assay, and normalized to the U6 levels; (B) Scatter plots of the Spearman correlation coefficient (rho) corresponding to an inverted monotonic trend between relative miR-21, and miR-34b, or miR-34c levels in breast cancer tumors; (C) Levels of miR-21, miR-34b and miR-34 in MCF-10A, T47D, MDA-MB-231, and MDA-MB-468 cells were determined by RT-qPCR assay, and normalized to the U6 levels; and (D) Serum levels of miR-21, miR-34b and miR-34 in breast cancer patients and healthy people were analyzed by Taqman RT-qPCR assay. All results represent the mean \pm SD from three independent experiments. ** indicate significant difference at $p<0.01$, respectively.

\subsection{Functions of MiR-21 and MiR-34b/c in Breast Cancer Cells}

To investigate the biological functions of miR-21, miR-34b, and miR-34c in BC cells, we used lentiviral vectors to establish T47D and MDA-MB-231 cell lines stably expressing non-sense sequence miR-NS, anti-miR-21, miR-34b/c, or triple expressing anti-miR-21 and miR-34b/c. We used these four miRNAs-stably expressing BC cell lines to perform in vitro and in vivo experiments, including cell growth, colony formation, cell migration, and tumor growth. The results showed that down-regulation of miR-21 and/or up-regulation of miR-34b/c led to a reduction in cellular proliferation, and migratory capacity, and impaired the ability of colony formation of both BC cell lines (see Supplementary Figure S1). We next examined the role for miR-21 and miR-34b/c in vivo by subcutaneous injection of miRNAs-stable expressing cells into nude mice. Within 30 days after injection, we found that miR-21 inhibition and/or miR-34b/c restoration resulted in substantial reduction of tumor growth comparing 
with control groups (see Figure 2A). Notably, among all treatment groups, the triple miRNAs-expressing groups exhibited the strongest cell proliferation, colony formation, cell migration, and tumor growth inhibition effect on BC cell lines.

To explore the mechanism of growth inhibition induced by anti-miR-21, and/or miR-34/c in BC, we searched a list of published or potential targets of miR-21 and miR-34b/c. We identified PTEN, a well known target of miR-21, and CDK4/6, two important targeted of miR-34b/c [13,14]. These proteins were crucial in miR-21- and miR-34b/c-mediated BC cellular function. We first evaluated the expression levels of PTEN, CDK4 and CDK6 in ten pairs of BC specimens by Western blot and immunohistochemical (IHC) staining. The results revealed that the expression of PTEN was down-regulated, and both $\mathrm{CDK} 4$ and CDK6 expression were up-regulated in breast tumor tissues and cancer cell lines compared with non-malignant adjacent tissues and MCF-10A, respectively (see Figure 2B and Supplementary Figure S2). Overexpression of anti-miR-21 increased PTEN expression, and restoration of miR-34b/c expression repressed CDK4/6 expression in BC cell lines (see Figure 2C).

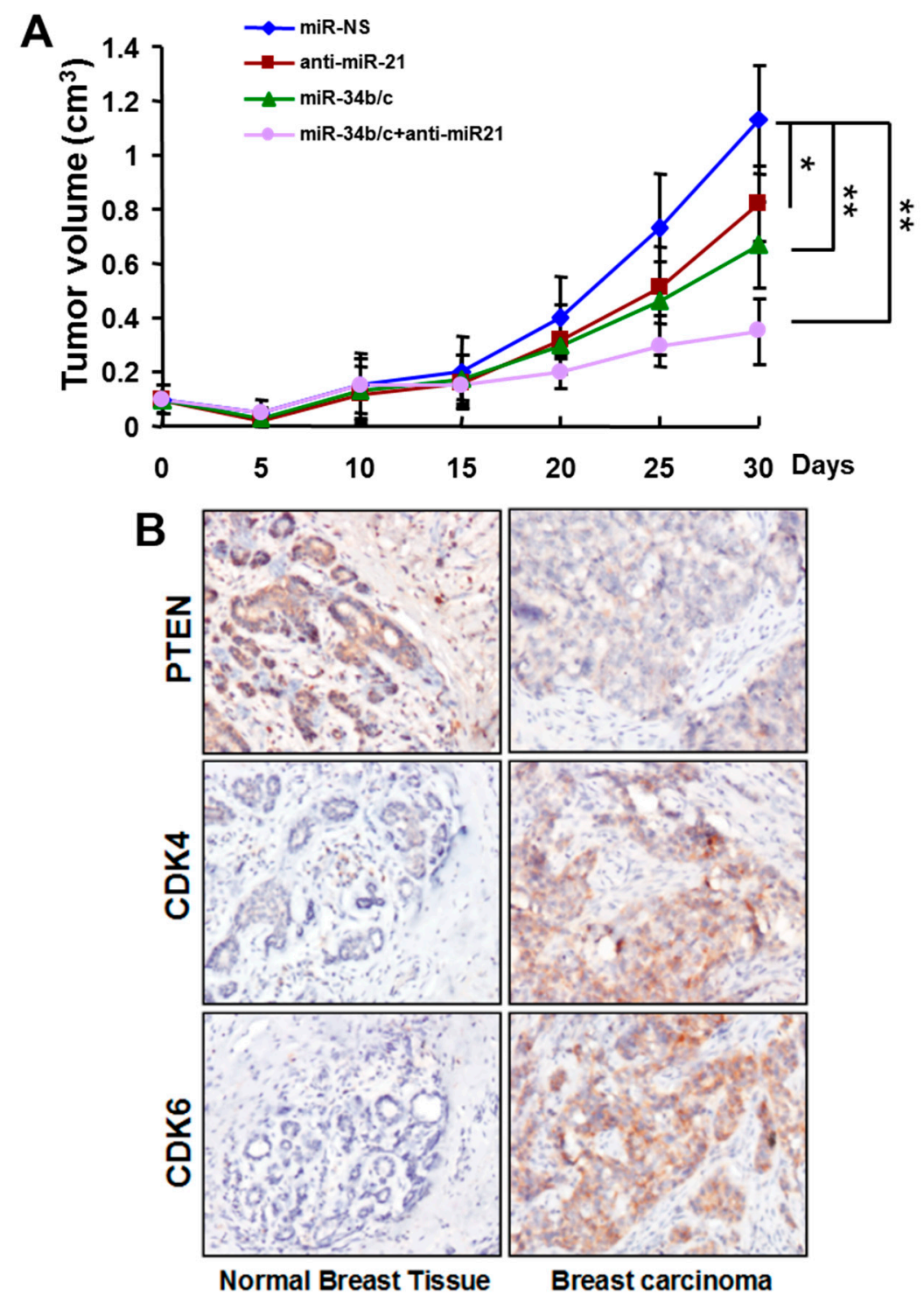

Figure 2. Cont. 


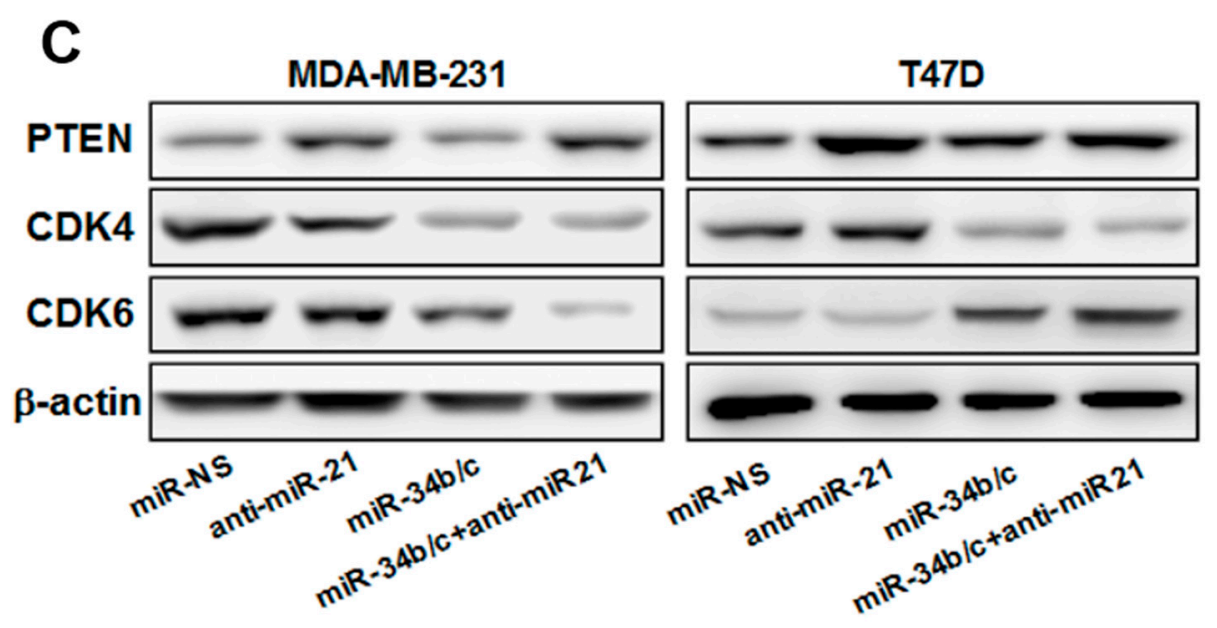

Figure 2. Functional roles of miR-21 and miR-34b/c in breast cancer. (A) MDA-MB-231 cells stable-expressing non-sense sequence (NS), anti-miR-21 and/or miR-34b/c were subcutaneously injected into immunodeficient mice. Tumor volumes were measured every five days. Xenografts were removed 30 days after implantation. Tumor volumes were obtained and presented (mean $\pm \mathrm{SEM} ; n=8$ ). $* p<0.05$, ** $p<0.01$; (B) Immunohistochemical (IHC) staining of PTEN, CDK4, and CDK6 in representative breast carcinoma tumors and non-malignant normal adjacent tissues. ( $\times 200$ magnification); and (C) T47D and MDA-MB-231 cells were infected with lentivirus carrying non-sense sequence (NS), anti-miR-21 and/or miR-34b/c. The expression levels of PTEN, CDK4, and CDK6 in these cells were analyzed by Western blot.

\subsection{MiR-21 Regulates MiR-34b/c Expression via PTEN/AKT/FOXO3a Signaling Pathway}

Interestingly, we found that miR-21 overexpression decreased the expression levels of miR-34b/c; while miR-21 knockdown enhanced miR-34b/c expression (see Figure 3A). To understand the molecular mechanism of how miR-21 regulates miR-34b/c expression, we searched for transcription factors involved in miR-34b/c expression. MiR-34b and miR-34c are processed from the same pre-miRNA (BC021736), indicating that miR-34b and miR-34c are regulated by the same transcription factor. FOXO3a was reported to be responsible for regulation of miR-34b/c expression [15]. To verify this conclusion in $\mathrm{BC}$ cell lines, we first constructed a luciferase reporter plasmid containing putative FOXO3a binding sites in the promoter region of BC021736. We observed that FOXO3a overexpression significantly enhanced the luciferase activity from the reporter construct (see Figure 3B). Chromatin immunoprecipitation-qPCR (ChIP-qPCR) results showed that the FOXO3a occupancy of the BC021736 promoter was markedly higher compared to the IgG background signals and negative control signals (see Figure 3C). The expression levels of miR-34b and miR-34c were enhanced by FOXO3a overexpression, but suppressed by siRNA-mediated knockdown of FOXO3a expression in MCF-10a cells (see Supplementary Figure S3A). Taken together, these results suggest that miR-34b and miR-34c are regulated by FOXO3a at transcriptional levels. We observed that forced expression of FOXO3a in miR-21 stable-expressing cell lines abolished miR-21-treated miR-34b/c down-regulation (see Supplementary Figure S3B), indicating FOXO3a is involved in miR-21 regulated miR-34b/c expression. 
Given that phosphorylation of FOXO3a by oncogenic kinases, such as AKT or ERK results in FOXO3a translocation from nucleus to cytoplasm and subsequent degradation [16], and PTEN is a well-characterized AKT pathway suppressor, we hypothesized that miR-21 regulated the activity of FOXO3a through the AKT/PTEN signaling pathway. To verify this hypothesis, MDA-MB-231 $(\mathrm{PTEN}+/+)$ and MDA-MB-468 (PTEN-/-) cells were transfected with anti-miR-Scr or anti-miR-21. We found that suppressing miR-21 enhanced levels of PTEN and nuclear levels of FOXO3a, and decreased levels of p-AKT, p-s253-FoxO3a and p-s315-FoxO3a in MDA-MB-231 with wild-type PTEN expression, but not in MDA-MB-468 with PTEN depletion (see Figure 3D). MiR-21 knockdown induced nuclear FOXO3a up-regulation leading to a robust increase of FOXO3a occupancy at the promoter of BC021736 in MDA-MB-231 cells (see Figure 3C). Moreover, forced expression of PTEN in MDA-MB-468 can mimic the effect of knockdown of miR-21 in MDA-MB-231 cell (see Figure 3E,F). Taken together, our results suggested that miR-21 regulates miR-34b/c expression via the PTEN/AKT/FOXO3a signaling pathway.

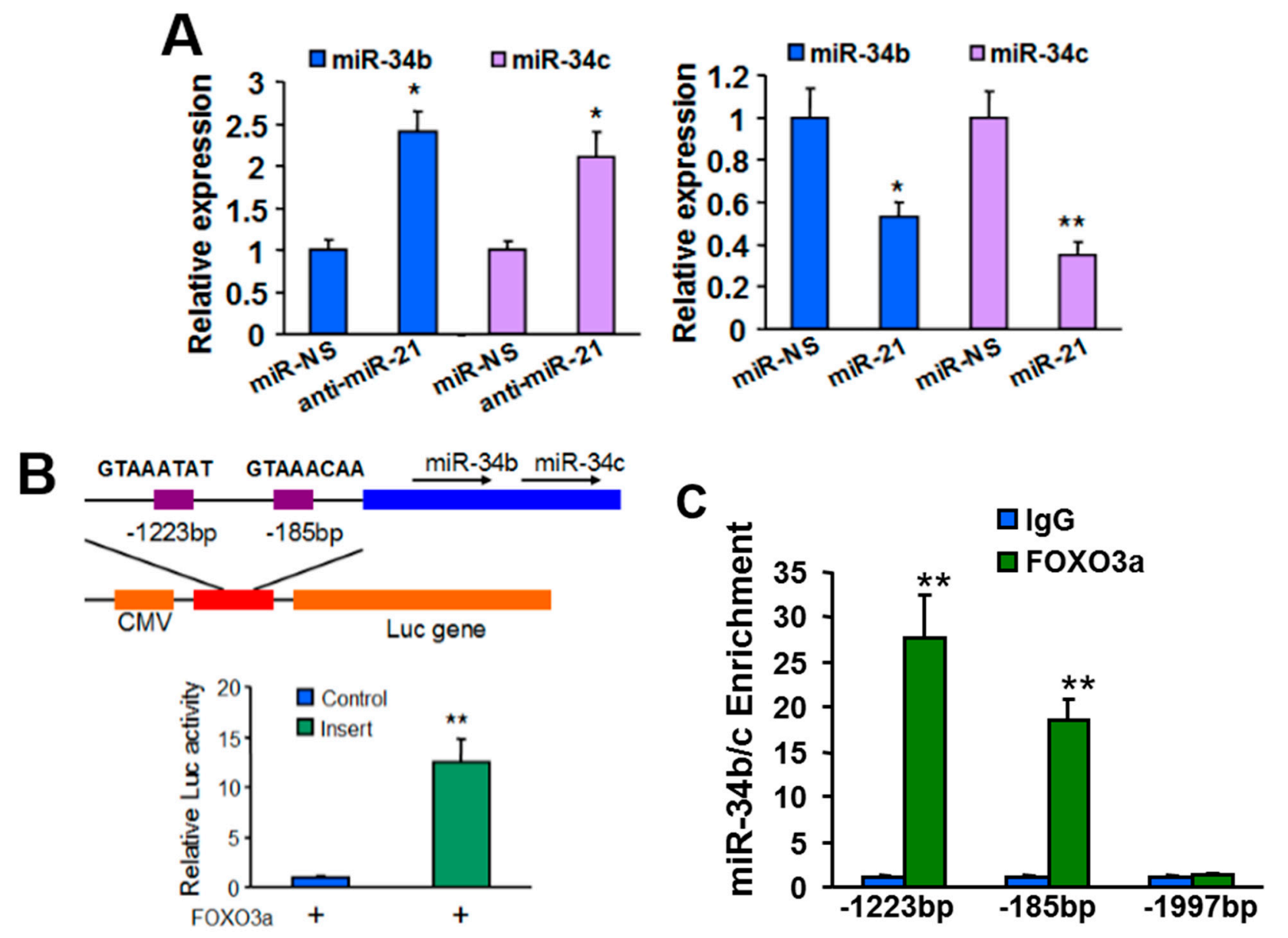

Figure 3. Cont. 


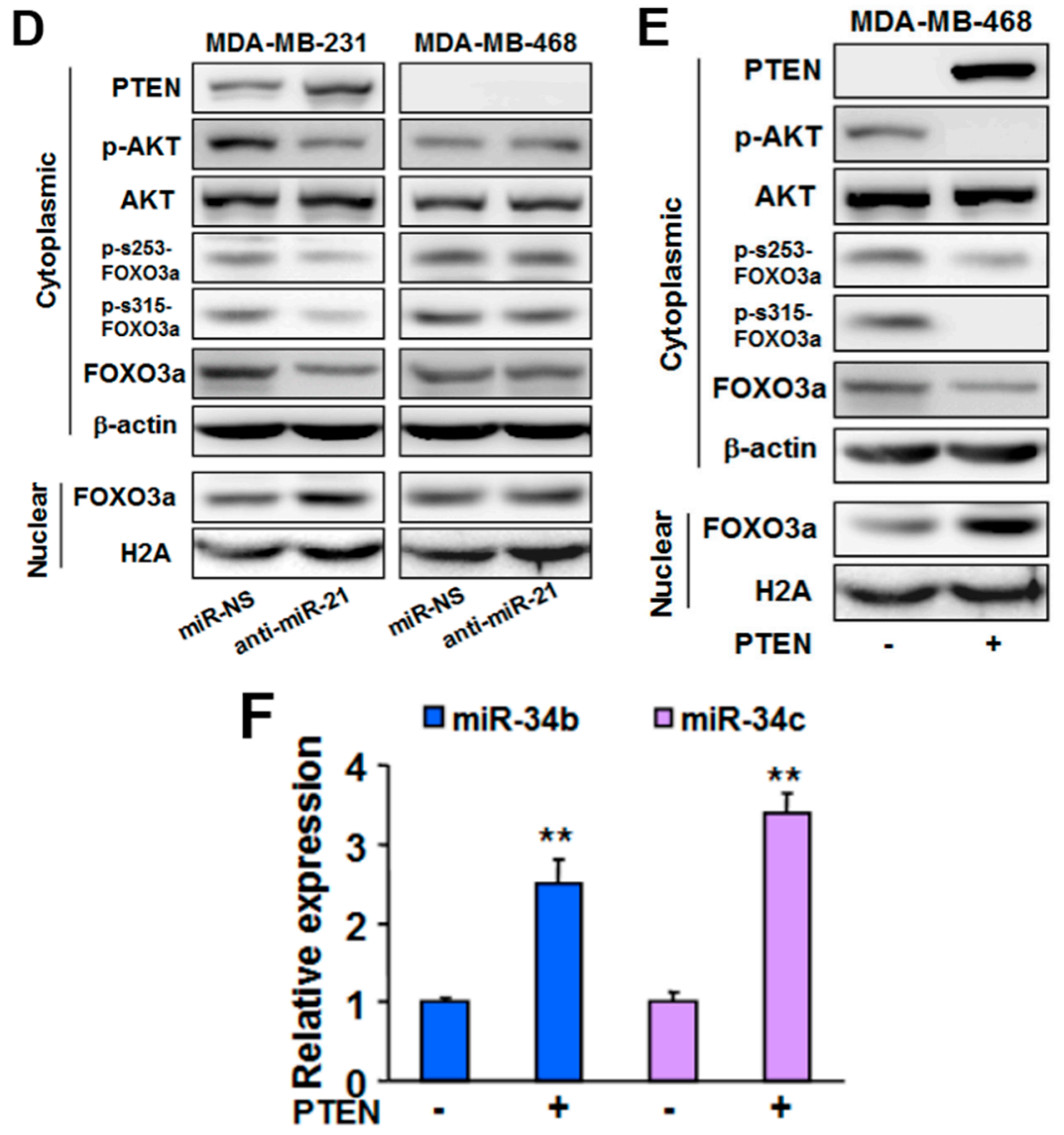

Figure 3. MiR-21 regulated miR-34b/c expression through AKT/PTEN/FOXO3a signaling. (A) MCF-10A cells were transfected with miR-NS, miR-21, or anti-miR-21 for $24 \mathrm{~h}$. The expression levels of miR-34b and miR-34c were analyzed by RT-qPCR and normalized to the U6 levels; (B) Schematic diagrams show the potential FOXO3a binding sites of the miR-34b/c promoter and the construction of miR-34b/c promoter luciferase reporter. Boxes indicate the distribution of two putative FOXO3a binding sites. Sequences of the consensus FOXO3a-binding sites are also shown. The reporter plasmid was co-transfected with FOXO3a expression plasmid and $\beta$-gal plasmid. The luciferase activities were normalized to the activity of $\beta$-gal, and values shown are normalized to the value of pGL-3 basic construct; (C) MCF-10A cells were infected with lentivirus carrying FOXO3a or empty vector (Control). Cross-linked chromatin was immunoprecipitated with antibodies specific for FOXO3a and IgG. Purified DNA was analyzed by RT-qPCR with three specific primer pairs as indicated in the Figure. The primers located in $-1997 \mathrm{bp}$ were used as a negative control; (D) MDA-MB-231 and MDA-MB-468 cells were infected with lentivirus carrying anti-miR-21 or miR-NS. Western blot analysis of nuclear levels of FOXO3a and cytoplasmic levels of PTEN, p-AKT, AKT, p-s253-FOXO3a, and p-s315-FOXO3a, respectively. $\mathrm{H} 2 \mathrm{~A}$ and $\beta$-actin were used as loading control; and (E,F) MDA-MB-468 cells were infected with lentivirus carrying PTEN or non-sense sequence. Western blot analysis of nuclear levels of FOXO3a and cytoplasmic levels of PTEN, p-AKT, AKT, p-s253-FOXO3a, and p-s315-FOXO3a in these cells, respectively, $(\mathbf{E})$ or RT-qPCR analysis of miR-34b and miR-34c levels $(\mathbf{F})$. Experiments were performed in triplicate and each was repeated three times. * $p<0.05, * * p<0.01$. 


\subsection{Systemic Delivery of MiR-34b/c Inhibits the Growth of Breast Tumors}

To investigate the therapeutic effect of miR-21 and/or miR-34b/c on breast tumors, we administrated formulated miRNAs by intravenous (i.v.) tail vein injection to BALB/c athymic nude mice bearing pre-established tumors. MDA-MB-231 cells were subcutaneously injected into the lower back of nude mice. On day five after xenograft implantation, tumor-bearing mice received i.v. tail vein injection of formulated miR-Scr, anti-miR-21, miR-34b/c, or anti-miR-21 and miR-34b/c combination. Each dose contained $20 \mu \mathrm{g}$ of miRNAs, which equals $1 \mathrm{mg} / \mathrm{kg}$ per mouse. Mice received formulated miRNAs injection every five days for a total of five times, and tumor volumes were measured each time before injection. As shown in Figure 4A, i.v. delivery of miR-34b/c, or anti-miR-21 and miR-34b/c combination dramatically reduced breast tumor growth in mice, and triple miRNAs delivery resulted in the greatest tumor growth suppression. However, the different tumor weight between anti-miR-21 injection and miR-Scr injection did not reach statistical significance in this study. These results indicate that targeting miR-21 and/or restoration of miR-34b/c exerts potential therapeutic efficacy against breast tumors.

\subsection{Discussion}

\subsubsection{MiR-21 and MiR-34b/c Expression in Breast Cancer}

A large-scale study of 540 human samples firstly demonstrated that miR-21 is the only miRNA that is up-regulated in six human cancers [17]. Years of studies demonstrated that the miR-34 family is down-regulated in most cancer types studied. Consistent with these observations, in the present study, we found that miR-21 was over-expressed and miR-34b/c was decreased in breast carcinoma of a Chinese population. Because serum or plasma samples are more feasible than tumor tissues in clinical practice, circulating miRNAs are highlighted as potential diagnostic markers for human cancer. Circulating miR-21 and miR-34a were reported to be up-regulated and down-regulated in breast cancer patients $[18,19]$, respectively, however, there has been no information related to circulating miR-34b and miR-34c in breast cancer patients. Our findings not only confirmed that serum levels of miR-21 in breast cancer patients are higher than in healthy controls, but also showed for the first time that circulating miR-34b and miR-34c were significantly down-regulated in serum from breast cancer patients, indicating that miR-21 and miR-34b/c are candidate serum biomarkers for breast cancer. 


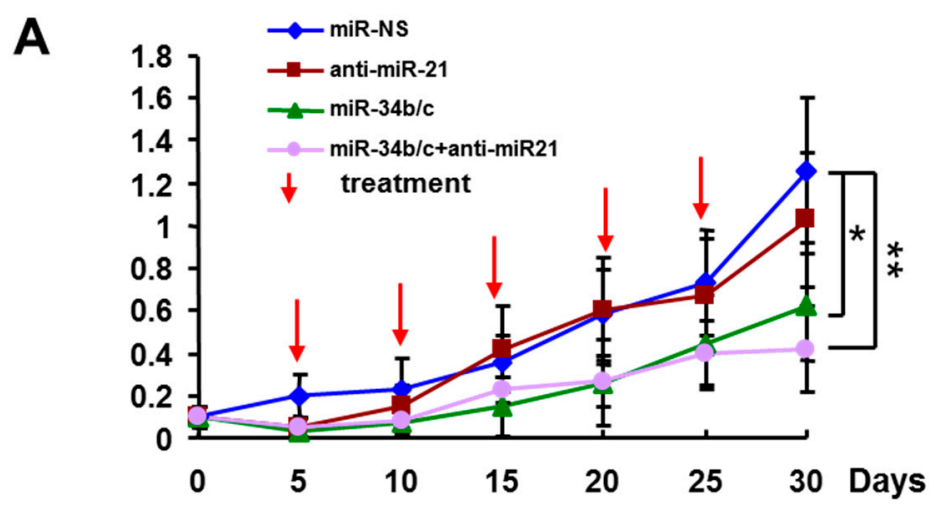

B

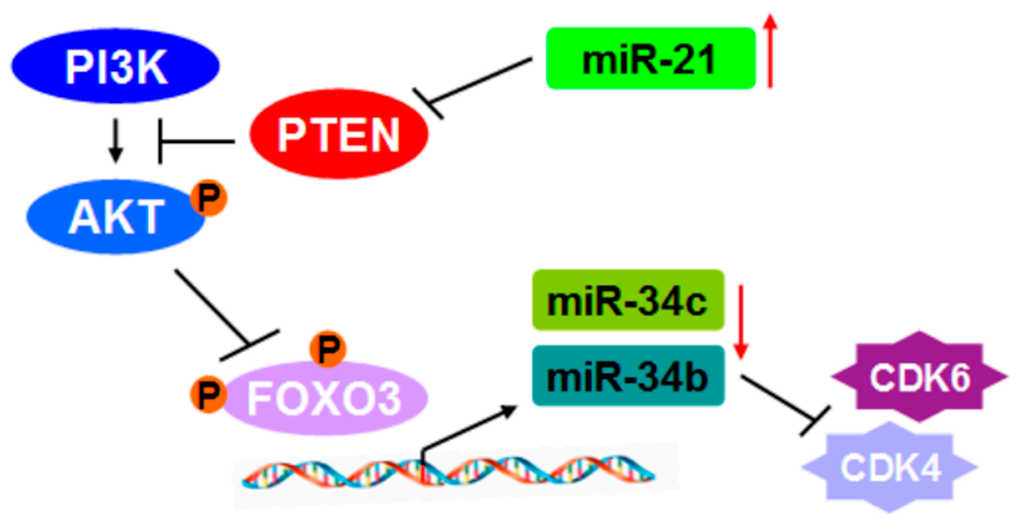

Figure 4. Systematic delivery of $\mathrm{miR}-34 \mathrm{~b} / \mathrm{c}$ or with anti-miR-21 inhibited breast tumor growth. (A) MDA-MB-231 cells were subcutaneously injected into immunodeficient mice. Tumor volumes were measured every five days. At day five after tumor implantation, the mice were treated with formulated miR-Scr, anti-miR-21, miR-34b/c, or anti-miR-21 and miR-34b/c combination by intravenous (i.v.) tail vein every five days. Tumor volumes were measured every five days and presented. (mean \pm SEM; $n=8$ ). ${ }^{*} p<0.05, * * p<0.01$; and (B) A model of the miR-21-FOXO3a-miR-34b/c signaling in breast cancer. Oncogenic miR-21 up-regulation promoted PI3K/AKT signaling activation through directly inhibiting PTEN expression, a suppressor of PI3K/AKT. The activation of AKT phosphorylates FOXO3a resulting in relocalization of $\mathrm{FOXO} 3 \mathrm{a}$ proteins from nucleus to the cytoplasm. Nuclear FOXO3a down-regulation reduced the binding efficiency of FOXO3a in the promoter of miR-34b/c leading to decreases in the expression levels of miR-34b and miR-34c in cells.

\subsubsection{Mechanism of MiR-21 Regulation of MiR-34b/c Expression}

It has been reported that the miR-34 family is regulated by p53 in p53 wild-type cells. In breast cancer cell lines (MDA-MB-231, MDA-MB-468, and T47D), the expression of miR-34b/c is independent of $\mathrm{p} 53$ regulation due to the inactivating mutations of $\mathrm{p} 53$. We found that miR-21 levels were inversely correlated with miR-34b and miR-34c levels, and induction of miR-34b/c in response to miR-21 down-regulation occur in breast cancer cells raising the possibility that miR-34b/c is regulated by other transcription factor in cancer cells. AKT phosphorylates FOXO3a triggering the rapid relocalization of FOXO3a proteins from nucleus to the cytoplasm, leading to repression of FOXO3a transcriptional activity [20,21]. We showed that miR-21 inhibition led to enhancing PTEN expression, 
and the latter inhibited the activity of AKT. Dephosphorylation of FOXO3a due to AKT inactivation, promotes FOXO3a nuclear translocation, resulting in enhanced FOXO3a-driven miR-34b/c upregulation. Although it has been shown that miRNAs can regulate other miRNAs via a direct interaction [22], we propose that miRNA-mediated miRNA expression through affecting transcription factor activity is more prevalent than miRNA-miRNA interaction in cells, since signal transduction has greater flexibility in regulation than the restrict requirements of miRNAs sequence binding.

\subsubsection{Functions of MiR-21 and MiR-34b/c in Breast Cancer}

It is well established that miR-21 acts as an oncogene and miR-34b/c as tumor suppressors in human cancer [23,24]. Recently, miRNAs have emerged as therapeutic options to treat cancer. The advantages of miRNAs-based treatment are obvious. Firstly, miRNAs can regulate a broad set of genes simultaneously. Secondly, miRNAs can also affect tumor-promoting stromal cells to modulate the tumor microenvironment. Thirdly, miRNAs showed reduced immune response and low toxicity when compared with lentivirus- or protein-based gene therapy [25]. The effect of systemic delivery of anti-miR-21 and/or miR-34b/c on breast tumors has never been evaluated. Our results demonstrate that co-injection of miR-34b/c with anti-miR-21 achieved stronger anti-tumor effect than miR-34b/c or anti-miR-21 alone-treatment. This finding further supports the notion that application of miRNAs as a therapeutic option for cancer treatment, and multiple miRNAs administration may elicit better therapeutic effect than single miRNA. Further studies focusing on improving miRNAs stability and delivery efficiency are crucial for clinical use of miRNA-based therapy.

\section{Experimental Section}

\subsection{Antibodies, Cell Lines, and Reagents}

Antibodies were as follows: PTEN, CDK4, CDK6, AKT, p-AKT, FOXO3a, p-s253-FOXO3a, and p-s315-FOXO3a were purchased from Cell Signaling Technology (Beverly, MA, USA). $\beta$-actin and H2A were obtained from Santa Cruz Biotechnonlgy (Santa Cruz, CA, USA). siRNA-targeting FOXO3a oligonucleotides was purchased from Thermo Scientific (Hudson, NH, USA) as specific oligo pools. Lentiviral plasmids expressing FOXO3a and PTEN were obtained from GeneCopoeia (Rockville, MD, USA), and anti-miR-21, miR-34b, and miR-34c were from (Open Biosystems, Pittsburgh, PA, USA), respectively. MCF-10A, T47D, MDA-MB-231 and MDA-MB-468 cells were purchased from ATCC (American Type Culture Collection, Manassas, VA, USA). Cells were maintained in $5 \% \mathrm{CO}_{2}$ incubator at $37^{\circ} \mathrm{C}$.

\subsection{Patients and Tissues Samples}

A total of 15 pairs of breast carcinoma tumors and adjacent normal tissues were obtained from Xiangya Hospital, Changsha, China. None of these patients received preoperative chemotherapy or radiotherapy before the surgery. EDTA-Blood samples were collected from healthy donors or breast cancer patients and processed for plasma isolation immediately. All samples were frozen and stored at $-80{ }^{\circ} \mathrm{C}$. Written informed consents were obtained from all patients in this study. This study 
was approved by the Ethics Committee of the Medical College of Central South University and Xiangya Hospital.

\subsection{Quantitative RT-PCR}

The expression levels of miRNAs were analyzed using Taqman MicroRNA Assay Kits (Applied Biosystems, Foster City, CA, USA) specific for hsa-miR-21, hsa-miR-34b and has-miR-34c. All experiments were performed in triplicate.

\subsection{Cell Migration Assay}

MDA-MB-231 or T47D cells stably-expressing non-sense sequence (NS), anti-miR-21 and/or $\mathrm{miR}-34 \mathrm{~b} / \mathrm{c}$ as indicated were plated in the top chamber. After incubation, the top chambers were wiped with cotton wool to remove the non-migratory cells. The exterior sides were fixed with $100 \%$ cold methanol, stained with $0.1 \%$ crystal violet, and air-dried. Cells were counted under a microscope.

\subsection{Immunohistochemistry Assay}

IHC was performed as previously described [26]. Tumor samples were fixed and processed by the paraffin-embedded method. Tissue sections were deparaffinized in xylene, and rehydrated in a graded ethanol series. After blocking with 5\% BSA, slides were probed overnight at $4{ }^{\circ} \mathrm{C}$ with monoclonal antibody against CDK4, CKD6 or PTEN. Primary antibody was detected by biotinylated secondary antibody followed by avidin-biotin peroxidase complex and 3,3'-diaminobenzidine chromagene (Life Technologies, Gaithersburg, MD, USA).

\subsection{Chromatin Immunoprecipitation-qPCR (ChIP-qPCR)}

ChIP-qPCR assays were performed using ChIP Assay Kit (Qiagen, Chatsworth, CA, USA). Purified DNA immunoprecipitated by FOXO3a or IgG antibody was used as a template for quantitative-PCR analysis. Primer sequences used in this study are listed as following: -185 Fwd (Forward) 5'-AATCA CCAAAAGCCCAGAAA-3', -185 Rev (Reverse) 5'-TTTTAAATGCCCACACAGAGG-3'; -1223 Fwd 5'-CCAAGATATGGTTTTATATTTCCTATGAG-3', -1223 Rev 5'-CTTGGCCTCTCACAGTGCTA-3'; -1997 Fwd 5'-CTGTAATCCCAGCTACTCA-3', -1997 Rev 5'-TTGGCTCACTGCAACCTC-3'.

\subsection{Mouse Xenograft Models}

$\mathrm{BALB} / \mathrm{c}$ athymic nude mice were housed and maintained in a laminar airflow cabinet in a pathogen-free environment. MDA-MB-231 cells $\left(1 \times 10^{6}\right)$ stably-expressing miR-NS, anti-miR-21, miR-34b/c, or miR-34b/c + anti-miR-21, were mixed with Growth Factor Reduced Matrigel (BD Lifesciences, Maryland, MD, USA) and injected subcutaneously into the lower back of each animal. Thirty days after injection, the mice were sacrificed by euthanasia and primary tumors were harvested from mice.

For miRNAs injection treatment, MDA-MB-231 cells $\left(1 \times 10^{6}\right)$ were mixed with Growth Factor Reduced Matrigel and injected subcutaneously into the right flank of each animal. miRNAs were formulated with MaxSuppressor in vivo RNALancerII (Bioo Scientific, Austin, TX, USA) according to the manufacturer's instructions. Each dose contained $20 \mu \mathrm{g}$ of formulated oligo, which equals $1 \mathrm{mg} / \mathrm{kg}$ 
per mouse with an average weight of $20 \mathrm{~g}$. Formulated miRNAs were intravenously (i.v.) by tail vein injected every 5 days starting on day 5 after tumor cells implantation.

\subsection{Statistical Analysis}

All results were analyzed using SPSS for windows version 13 (SPSS, Chicago, IL, USA). Quantitative variables were analyzed by $t$-test or ANOVA. The correlations were analyzed using Spearman's rank test. Differences were considered significant with $p$ less than 0.05 .

\section{Supplementary Materials}

Supplementary materials can be found at http://www.mdpi.com/1422-0067/16/02/3148/s1.

\section{Acknowledgments}

This work was supported by the Research Fund for Young Scholars of China (81101654).

\section{Author Contributions}

Xiangyan Liu, Jie Feng, Liqiu Liao and Shaihong Zhu conceived and designed the experiments; Xiangyan Liu, and Jie Feng performed the experiments; Lili Tang, Xiangyan Liu, Liqiu Liao and Jie Feng analyzed the data; Qing $\mathrm{Xu}$, Jie Feng, and Shaihong Zhu contributed to the writing of the manuscript. All authors read and approved the final manuscript.

\section{Conflicts of Interest}

The authors declare no conflict of interest.

\section{References}

1. Siegel, R.; Naishadham, D.; Jemal, A. Cancer statistics, 2013. CA Cancer J. Clin. 2013, 63, 11-30.

2. Radojicic, J.; Zaravinos, A.; Vrekoussis, T.; Kafousi, M.; Spandidos, DA.; Stathopoulos, E.N. MicroRNA expression analysis in triple-negative (ER, PR and Her2/neu) breast cancer. Cell Cycle 2011, 10, 507-517.

3. Sempere, LF.; Christensen, M.; Silahtaroglu, A.; Bak, M.; Heath, C.V.; Schwartz, G.; Wells, W.; Kauppinen, S.; Cole, C.N. Altered microRNA expression confined to specific epithelial cell subpopulations in breast cancer. Cancer Res. 2007, 67, 11612-11620.

4. Sieuwerts, A.M.; Mostert, B.; Vires, J.B.; Peeters, D.; de Jongh, F.; Stouthard, J.; van Galen, A.; Dirix, L.Y.; van Dam, P.A.; de Weerd, V.; et al. mRNA and microRNA expression profiles in circulating tumor cells and primary tumors of metastatic breast cancer patients. Clin. Cancer Res. 2011, 17, 3600-3618.

5. Iorio, M.V.; Ferracin, M.; Liu, C.G.; Veronese, A.; Spizzo, R.; Sabbioni, S.; Magri, E.; Pedriali, M.; Fabbri, M.; Campiglio, M.; et al. MicroRNA gene expression deregulation in human breast cancer. Cancer Res. 2005, 65, 7065-7070. 
6. Wickramasinghe, N.S.; Manavalan, T.T.; Dougherty, S.M.; Riggs, K.A.; Li, Y.; Klinge, C.M. Estradiol downregulates miR-21 expression and increases miR-21 target gene expression in MCF-7 breast cancer cells. Nucleic Acids Res. 2009, 37, 2584-2595.

7. He, L.; He, X.; Lim, L.P.; de Stanchina, E.; Xuan, Z.; Liang, Y.; Xue, W.; Zender, L.; Magnus, J.; Ridzon, D.; et al. A microRNA component of the p53 tumour suppressor network. Nature 2007, 447, 1130-1134.

8. Li, Y.; Guessous, F.; Zhang, Y.; Dipierro, C.; Kefas, B.; Johnson, E.; Marcinkiewicz, L.; Jiang, J.; Yang, Y.; Schmittgen, T.D.; et al. MicroRNA-34a inhibits glioblastoma growth by targeting multiple oncogenes. Cancer Res. 2009, 69, 7569-7576.

9. Yang, S.; Li, Y.; Gao, J.; Zhang, T.; Li, S.; Luo, A.; Chen, H.; Ding, F.; Wang, X.; Liu, Z. MicroRNA-34 suppresses breast cancer invasion and metastasis by directly targeting Fra-1. Oncogene 2013, 32, 4294-4303.

10. Yamakuchi, M.; Ferlito, M.; Lowenstein, C.J. miR-34a repression of SIRT1 regulates apoptosis. Proc. Natl. Acad. Sci. USA 2008, 105, 13421-13426.

11. Hermeking, H. The miR-34 family in cancer and apoptosis. Cell Death Differ. 2010, 17, 193-199.

12. Trang, P.; Wiggins, J.F.; Daige, C.L.; Cho, C.; Omotola, M.; Brown, D.; Weidhaas, J.B.; Bader, A.G.; Slack, F.J. Systemic delivery of tumor suppressor microRNA mimics using a neutral lipid emulsion inhibits lung tumors in mice. Mol. Ther. 2011, 19, 1116-1122.

13. Bommer. G.T.; Gerin. I.; Feng, Y.; Kaczorowski. A.J.; Kuick. R.; Love, R.E.; Zhai, Y.; Giordano. T.J.; Qin. Z.S.; Moore B.B.; et al. p53-Mediated activation of miRNA34 candidate tumor-suppressor genes. Curr. Biol. 2007, 17, 1298-1307.

14. Sun, F.; Fu, H.; Liu, Q.; Tie, Y.; Zhu, J.; Xing, R.; Sun, Z.; Zheng, X. Downregulation of CCND1 and CDK6 by miR-34a induces cell cycle arrest. FEBS Lett. 2008, 582, 1564-1568.

15. Kress, T.R.; Cannell, I.G.; Brenkman, A.B.; Samans, B.; Gaestel, M.; Roepman, P.; Burgering, B.M.; Bushell, M.; Rosenwald, A.; Eilers, M. The MK5/PRAK kinase and Myc form a negative feedback loop that is disrupted during colorectal tumorigenesis. Mol. Cell. 2011, 41, 445-457.

16. Yang, J.Y.; Hung, M.C. A new fork for clinical application: Targeting forkhead transcription factors in cancer. Clin. Cancer Res. 2009, 15, 752-757.

17. Volinia, S.; Calin, G.A.; Liu, C.G.; Ambs, S.; Cimmino, A.; Petrocca, F.; Visone, R.; Iorio, M.; Roldo, C.; Ferracin, M.; et al. A microRNA expression signature of human solid tumors defines cancer gene targets. Proc. Natl. Acad. Sci. USA 2006, 103, 2257-2261.

18. Eichelser, C.; Flesch-Janys, D.; Chang-Claude, J.; Pantel, K.; Schwarzenbach, H. Deregulated serum concentrations of circulating cell-free microRNAs miR-17, miR-34a, miR-155, and miR-373 in human breast cancer development and progression. Clin. Chem. 2013, 10, 1489-1496.

19. Wang, F.; Zheng, Z.; Guo, J.; Ding, X. Correlation and quantitation of microRNA aberrant expression in tissues and sera from patients with breast tumor. Gynecol. Oncol. 2010, 3, 586-593.

20. Brunet, A.; Bonni, A.; Zigmond, M.J.; Lin, M.Z.; Juo, P.; Hu, L.S.; Anderson M.J.; Arden, K.C.; Blenis, J.; Greenberg, M.E. Akt promotes cell survival by phosphorylating and inhibiting a Forkhead transcription factor. Cell 1999, 96, 857-868.

21. Greer, E.L.; Brunet, A. FOXO transcription factors at the interface between longevity and tumor suppression. Oncogene 2005, 50, 7410-7425. 
22. Chen, P.S.; Su, J.L.; Cha, S.T.; Tarn, W.Y.; Wang, M.Y.; Hsu, H.C.; Lin, M.T.; Chu, C.Y.; Hua, K.T.; Chen, C.N.; et al. miR-107 promotes tumor progression by targeting the let-7 microRNA in mice and humans. J. Clin. Investig. 2011, 9, 3442-3455.

23. Lu, Z.; Liu, M.; Stribinskis, V.; Klinge, C.M.; Ramos, K.S.; Colburn, N.H.; Li, Y. MicroRNA-21 promotes cell transformation by targeting the programmed cell death 4 gene. Oncogene 2008, 31 , 4373-4379.

24. Papagiannakopoulos, T.; Shapiro, A.; Kosik, K.S. MicroRNA-21 targets a network of key tumor-suppressive pathways in glioblastoma cells. Cancer Res. 2008, 68, 8164-8172.

25. Wiggins, J.F.; Ruffino, L.; Kelnar, K.; Omotola, M.; Patrawala, L.; Brown, D.; Bader, A.G. Development of a lung cancer therapeutic based on the tumor suppressor microRNA-34. Cancer Res. 2010, 70, 5923-5930.

26. Xu, Q.; Jiang, Y.; Yin, Y.; Li, Q.; He, J.; Jing, Y.; Qi, Y.T.; Xu, Q.; Li, W.; Lu, B.; et al. A regulatory circuit of miR-148a/152 and DNMT1 in modulating cell transformation and tumor angiogenesis through IGF-IR and IRS1. J. Mol. Cell Biol. 2013, 5, 3-13.

(C) 2015 by the authors; licensee MDPI, Basel, Switzerland. This article is an open access article distributed under the terms and conditions of the Creative Commons Attribution license (http://creativecommons.org/licenses/by/4.0/). 